\section{Über Siedepunkts-Reductionstafeln} auf Normaldruck und Thermometer mit Temperatur und Spannkraftstheilungen von Dämpfen.

Von

Paul Fuchs.

Der Siedepunkt einer Flüssigkeit ist bekanntlich die Temperatur seines Dampfes, welche unter einem Druck von $760 \mathrm{~mm}$ Quecksilbersäule bei $0^{0}$ gemessen wird.

Aus den Untersuchungen Crafts' (Ber. deutsch. 20, 279) ergab sich, dass die Siedepunktsänderung $\Delta$ innerbalb njcht zu grosser Schwankungen des Luftdrucks diesem direct proportional der absoluten Siedetemperatur $T$ des Körpers angesehen werden kann.

Man erhält so

$$
\Delta=\mathrm{T} \cdot \mathrm{c},
$$

wo $c$ eine Constante bedeutet, die von der chemischen Natur des zur Untersuchung gelangenden Körpers abhängt.

Für verschiedene Substanzen ist die Kenntniss dieser Constanten von Wichtigkeit, so für Benzol, Aceton u.s. w., Körper, deren Reinheit und andere Merkmale aus der bei $760 \mathrm{~mm}$ Druck abgeleiteten Siedetemperatur bestimmt werden.

Die Bedingungen des Normaldrucks bei Siedepunktsbestimmungen sind nun in Laboratorien selten anzutreffen und man bat neben Aufstellung von Reductionstafeln auch $\mathrm{Me}-$ thoden ersonnen, um Resultate unabhängig vom herrschenden Luftdruck zu gewinnen und die weiter unten besprochen werden.

Für eine Anzahl von Substanzen finden sich die von verschiedenen Autoren experimentell ermittelten Werthe umgerechnet in der folgenden Tafel:

1. für die Siedepunktsänderung $\Delta$ pro $1 \mathrm{~mm}$ Druckänderung und

2. für die Spannkraftänderung $\boldsymbol{A}_{0}$ des Dampfes der Substanz pro $0,1^{\circ}$ Temperaturvariation.

\begin{tabular}{l|l|lr}
\hline & \multicolumn{1}{|c|}{$\Delta$} & \multicolumn{2}{|c}{$\Delta_{0}$} \\
\hline Aceton, $\left(\mathrm{CH}_{3}\right)_{2} \mathrm{CO}$ & $0,0388^{0}$ & $\left.2,57 \mathrm{~mm}^{1}\right)$ \\
Athylalkohol, $\mathrm{C}_{.,} \mathrm{H}_{5} . \mathrm{OH}$ & 0,0362 & 2,76 & $2)$ \\
Anilin, $\mathrm{C}_{6} \mathrm{H}_{5} \cdot \mathrm{N} \mathrm{H}_{2}$ & 0,0518 & $\mathbf{1 , 9 3}$ & $3)$ \\
Benzol, $\mathrm{C}_{6} \mathrm{H}_{6}$ & 0,0430 & 2,32 & $4)$ \\
Methylalkohol, $\mathrm{CH}_{4}$ & 0,0362 & 2,76 & $2)$ \\
Monobrombenzol, $\mathrm{C}_{6} \mathrm{H}_{5} \mathrm{Br}$ & 0,0526 & 1,90 & $3)$ \\
Monochlorbenzol, $\mathrm{C}_{6} \mathrm{H}_{5} \mathrm{Cl}$ & 0,0496 & 2,01 & $3)$ \\
Meta-Xylol, $\mathrm{C}_{6} \mathrm{H}_{4}\left(\mathrm{CH}_{3}\right)_{2}$ & 0,0508 & 1,96 & $1)$
\end{tabular}
8,628

1) Crafts, Ber. d. d. chem. Ges. 20, 409.

2) Schmidt, Ztschr. f. phys. Chem. 7, 433;

1, 247

3) Ramsay und Young, Ztschr. f. phys. Chemie

4) Regnault, Mémoire de l'acad. 21, 624; 26,

339. Compt. rend. 39, 301, 347, 397.

Ch. 98.
Diese Werthe haben Gültigkeit für eine Druckdifferenz von $\pm 50 \mathrm{~mm}$ gegenüber dem Normaldruck von $760 \mathrm{~mm}$ bei $0^{\circ}$.

Bedeutet $n$ die herrschende Druckabweichung vom Normaldruck $760 \mathrm{~mm} \mathrm{Hg-}$ Säule bei $0^{0}$, t die hierbei beobachtete Siedetemperatur, so ergibt sich der auf Normaldruck reducirte Siedepunkt der Substanz zu $\pm(n \cdot c) t$

welche Werthe in den am Schluss befindlichen Tafeln für obengenannte Substanzen zu finden sind.

Ausser der Benutzung von Siedepunktscorrectionstafeln hat man auch Thermometer mit verstellbarer Scale zur Reduction auf $760 \mathrm{~mm}$ Druck eingeführt. Der Gebrauch dieser Instrumente ist nicht empfehlenswerth.

So kann beispielsweise ein einmal eingestelltes Instrument leicht von unberufenen Händen verstellt werden, ein Übelstand, der ohne Weiteres gar nicht auffällt.

Ferner wird oft durch unzweckmässiges Schrauben die Capillare vom Gefäss abgesprengt und zuletzt sind die Angaben dieser Instrumente ihrer veränderlichen Scale halber für exacte Bestimmungen wenig vertrauenerweckend, so dass z. B. eine Prüfung dieser Thermometer von Seiten einer Behörde aus geschlossen ist.

Man kann diese Bedenken durch Construction folgender Thermometer eliminiren. Trägt man neben einer Temperaturscale, welche beispielsweise für Benzol bestimmt sei, die Spannkraft seines Dampfes bei verschiedenen Temperaturen auf, so hat man bei einem reinen Benzol aus einer Siedepunktsermittelung ohne Beobachtung des Luftdruckes die Möglichkeit, den bei $760 \mathrm{~mm}$ Druck bei $0^{0}$ zugehörigen Normalsiedepunkt direct abzulesen. Die Figur 200 zeigt ein Instrument für $\mathrm{C}_{6} \mathrm{H}_{6} ; \alpha$ ist eine Temperaturscale, angepasst dem Siedepunkte des Benzols, Gradumfang von 77,5 bis $81,0^{0}, \beta$ ist eine diesen Temperaturen entsprechende Spannkrafts- $\mathbf{I}$ Fig. 200. scale des Benzoldampfes.

Reines Benzol siedet unter normalen Verhältnissen bei $+80,0^{\circ}$. Würde man den Druck auf $730 \mathrm{~mm}$ erniedrigen, so wäre der Stand des Quecksilberfadens der Temperaturscale bei $78,71^{\circ}$, oder liesse man den Druck auf $780 \mathrm{~mm}$ steigen, könnte man den zugehörigen thermischen Werth zu $80,86^{\circ}$ ablesen. 
In deu seltensten Fällen will man nun Siedepunkte von anerkannt reinen Substanzen ermitteln, sondern diese Beobachtung soll ja meist einen Schluss auf die Reinheit des Präparates erst erlauben.

Hier hätte man zu der Beobachtung des Siedepunktes und des Dampfdruckes am Thermometer noch eine Ermittelung des beim Siedevorgang herrscheoden Luftdruckes zu machen.

Der auf $0^{0}$ reducirte Barometerstand würde sich mit dem auf der Spannkraftsscale abgelesenen decken, wenn ein reines Benzol vorlag. Liegt eine Substanz von anderem Siedepunkte vor, würde man eine Correctur anzubringen baben. Man erhalte beispielsweise folgende Resultate.

Versuchsobject: Benzol.

Thermometerablesungen in siedender Substanz:

Dampfdruck in mm Hg-Säule $79,28^{\circ} \cdot$. Barometerablesung, red. auf $0^{0} .746$. . $\gamma$
Man erhält eine Differenz $\gamma-\beta$ ron $3 \mathrm{~mm}$ im Dampfdruck des Benzols.

Der zu $760 \mathrm{~mm}$ zugehörige Siedepunkt ist in diesem Falle nicht neben $760 \mathrm{~mm}$, sondern neben $763 \mathrm{~mm}$ abzulesen, so dass man einen Normalsiedepunkt dieses Versuchsobjectes zu $80,13^{\circ}$ erhält.

Wird in der Differenz $\boldsymbol{\gamma}-\boldsymbol{\beta} \beta$ grösser als $\gamma$, so muss die Correctionsgrösse selbstverständlich negativ genommen werden.

Die Scalen dieser Thermometer mit Spannkraftstheilung von Dämpfen sind in $0,05^{\circ}$ getheilt und geben sehr genaue Resultate.

Durch die gegenseitige Beobachtung der Spannkraftstheilung des Dampfes auf dem Thermometer und des ermittelten Luftdrucks kann das Instrument bequem auf Febler controlirt werden; überhaupt gibt ein statisches Thermometer immer exacter zu controlirende Resultate als metastatische Instrumente, wozu die eingangs erwähnten mit verstellbaren Scalen gehören ${ }^{5}$ ).

Siedepunkts-Reductionstafel auf Normaldruck von $760 \mathrm{~mm}$ für Aceton, $\left(\mathrm{CH}_{3}\right)_{2} \mathrm{CO}$.

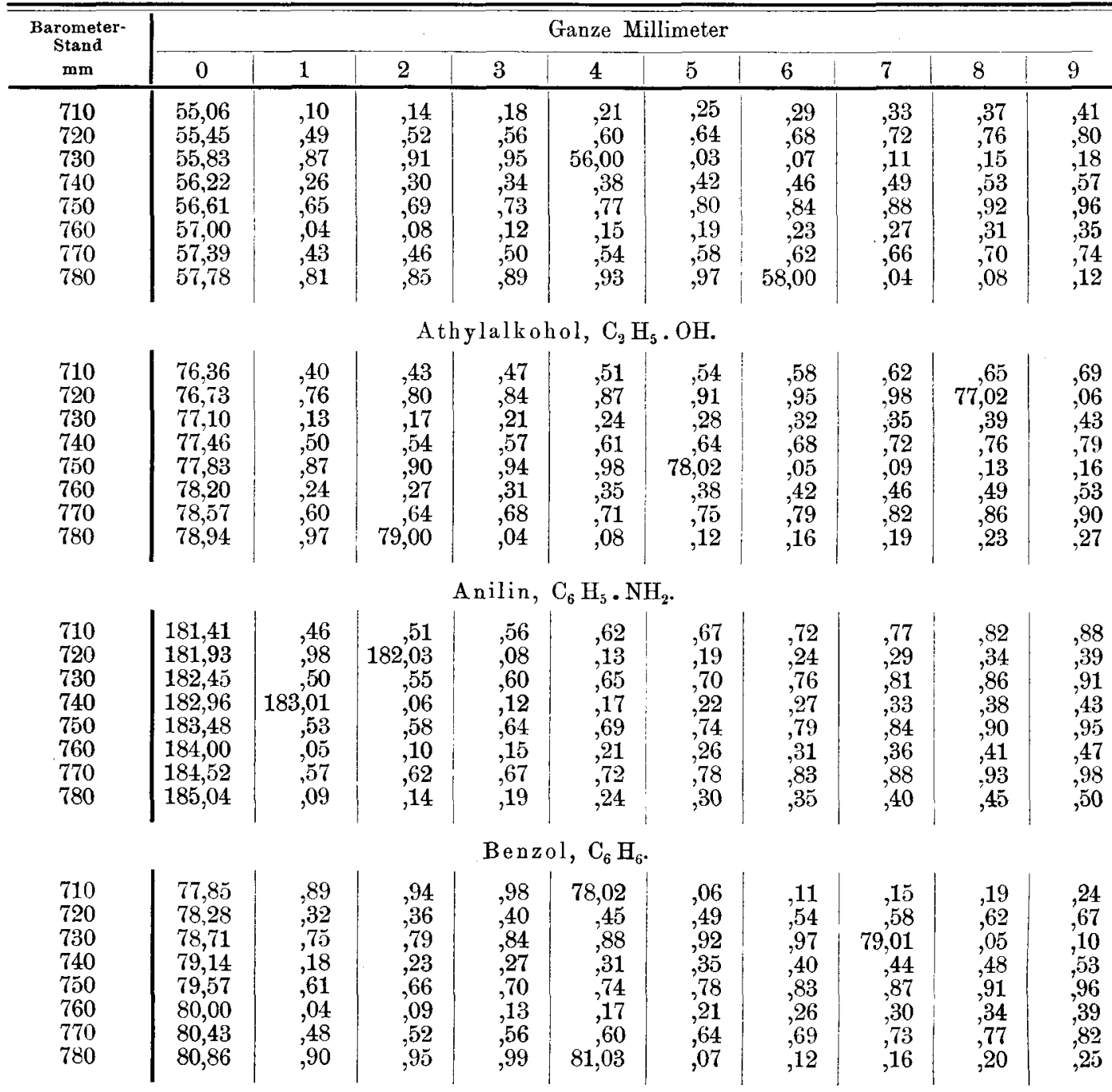

5) Das glastechnische Institut ron Gustav Müller-Jlmenau fertigt diese Instrumente. 
Siedepunkts-Reductionstafel auf Normaldruck von $760 \mathrm{~mm}$ für Methylalkohol, $\mathrm{CH}_{4}$.

\begin{tabular}{|c|c|c|c|c|c|c|c|c|c|c|}
\hline \multirow{2}{*}{$\begin{array}{l}\text { Barometer- } \\
\text { Stand } \\
\text { mm }\end{array}$} & \multicolumn{10}{|c|}{ Ganze Millimeter } \\
\hline & 0 & 1 & 2 & 3 & 4 & 5 & 6 & 7 & 8 & 9 \\
\hline 710 & 65,06 &, 10 &, 13 &, 17 &, 21 &, 24 &, 28 &, 32 &, 35 &, 39 \\
\hline 720 & 65,43 & 46 &, 50 &, 54 &, 57 &, 61 &, 65 & 68 &, 72 &, 76 \\
\hline 730 & 65,80 & 83 & .87 & 91 &, 94 & 98 & 66,02 &, 05 &, 09 & ,13 \\
\hline 740 & 66,16 & 20 & 24 & .27 & 31 &, 35 &, 38 &, 42 &, 46 &, 49 \\
\hline 750 & 66,53 & 57 &, 60 &, 64 &, 68 &, 72 &, 75 &, 79 &, 83 &, 86 \\
\hline 760 & 66,90 & 94 & .97 & 67,01 &, 05 &, 08 & ,12 &, 16 & ,19 & ,23 \\
\hline 770 & 67,27 &, 30 &, 34 &, 38 &, 42 &, 45 &, 49 &, 53 &, 56 & ,60 \\
\hline 780 & 67,64 &, 67 &, 71 &, 75 &, 78 &, 82 &, 86 & ,89 &, 93 &, 97 \\
\hline
\end{tabular}

Monobrombenzol, $\mathrm{C}_{6} \mathrm{H}_{5}$. Br.

\begin{tabular}{l|l|r|r|r|r|r|r|r|r|r}
710 & 153,37 &, 42 &, 47 &, 53 &, 58 &, 63 &, 68 &, 74 &, 79 &, 84 \\
720 & 153,90 &, 95 & 154,00 &, 05 &, 11 &, 16 &, 21 &, 26 &, 32 &, 37 \\
730 & 154,42 &, 47 &, 53 &, 58 &, 63 &, 68 &, 74 &, 79 &, 84 &, 89 \\
740 & 154,95 & 155,00 &, 05 &, 11 &, 16 &, 21 &, 26 &, 32 &, 37 &, 42 \\
750 & 155,47 &, 53 &, 58 &, 63 &, 68 &, 74 &, 79 &, 84 &, 89 &, 95 \\
760 & 156,00 &, 05 &, 10 &, 16 &, 21 &, 26 &, 32 &, 37 &, 42 &, 47 \\
770 & 156,53 &, 58 &, 63 &, 68 &, 74 &, 79 &, 84 &, 89 &, 95 & 157,00 \\
780 & 157,05 &, 10 &, 16 &, 21 &, 26 &, 31 &, 37 &, 42 &, 47 &, 03
\end{tabular}

\begin{tabular}{l|l|l|l|l|l|l|l|l|l|l|l}
710 & 129,52 &, 57 &, 62 &, 67 &, 72 &, 77 &, 82 &, 87 &, 92 &, 97 \\
720 & 130,02 &, 07 &, 12 &, 16 &, 21 &, 26 &, 31 &, 36 &, 41 &, 46 \\
730 & 130,51 &, 56 &, 61 &, 66 &, 71 &, 76 &, 81 &, 86 &, 91 &, 96 \\
740 & 131,00 &, 06 &, 11 &, 16 &, 21 &, 26 &, 31 &, 36 &, 40 &, 45 \\
750 & 131,50 &, 55 &, 60 &, 65 &, 70 &, 75 &, 80 &, 85 &, 90 &, 95 \\
760 & 132,00 &, 05 &, 10 &, 15 &, 20 &, 25 &, 30 &, 35 &, 40 &, 45 \\
770 & 132,50 &, 55 &, 60 &, 64 &, 69 &, 74 &, 79 &, 84 &, 89 &, 94 \\
780 & 132,99 & $\mathbf{1 3 3 , 0 4}$ &, 09 &, 14 &, 19 &, 24 &, 29 &, 34 &, 39 &, 44
\end{tabular}

\begin{tabular}{|c|c|c|c|c|c|c|c|c|c|c|}
\hline 710 & 136,46 &, 51 &, 56 &, 61 &, 66 &, 71 &, 76 &, 82 & 87 &, 92 \\
\hline 720 & 136,97 & 137,02 &, 07 &, 12 & 17 & ,22 &, 27 & ,32 & 37 &, 42 \\
\hline 730 & 137,48 &, 53 &, 58 & 63 &, 68 &, 73 & ,78 &, 83 & 88 &, 93 \\
\hline 740 & 137,98 & 138,03 & 09 & 14 & ,19 & ,24 &, 29 & ,34 & ,39 &, 44 \\
\hline 750 & 138,49 &, 54 &, 59 &, 64 &, 69 &, 75 & 80 &, 85 &, 90 &, 95 \\
\hline 760 & 139,00 & ,05 &, 10 & ,15 & ,20 & ,25 & ,30 &, 36 & ,41 &, 46 \\
\hline 770 & 139,51 &, 56 &, 61 & ,66 &, 71 & ,76 & ,81 &, 86 &, 91 &, 97 \\
\hline 780 & 140,02 &, 07 & ,12 & ,17 &, 22 & ,27 &, 32 &, 37 &, 42 &, 47 \\
\hline
\end{tabular}

Der dritte internationale Congress für angewandte Chemie.

[Schluss von S. 775.]

Von den sonstigen Verhandlungen der 1. Section ist der Vorschlag von A. Jolles zu erwähnen, zur Bestimmung der Phosphorsäure in Wasser die Probe, nach Abscheidung der Kieselsäure, mit Kaliummolybdat zu versetzen und die Färbung mit bekannten Lösungen zu vergleichen. Als Urtitersubstanz empfiehlt Sörensen das neutrale Natriumoxalat. -

Es mögen nun die ron den einzelnen Sectionen gefassten Beschlüsse folgen:

Section I. 1. Es sei zu erstreben, dass die Fabrikanten chemischer Präparate die Stoffe und ibre Quantitäten angeben, die als unver- meidliche "fremde" Bestandtheile in diesen "chemisch reinen" Präparaten enthalten sind.

Die Fachgenossen werden aufgefordert, die in dieser Hinsicht in der $\mathrm{Z}$ wischenzeit gemachten Erfahrungen dem Secretär des nāchsten Congresses möglichst umgehend mitzutheilen, welcher diese Mittheilungen einer Section für analytische Chemie zur weiteren Berathung zu übergeben hat.

2. Die Section beschliesst, das Organisations-Comité des nächsten Congresses zu ersuchen, die Frage einheitlicher Titersubstanzen auf die Tagesordnung zu setzen und auf möglichst weiter Basis recht bald vorzubereiten.

Section II. 1. Die Section II a des III. internationalen Congresses für angewandte Chemie spricht den Wunseh aus, dass die 